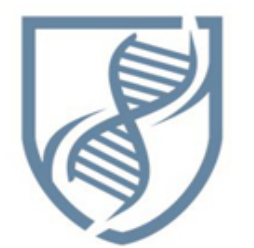

Journal of Bioscience and Applied Research

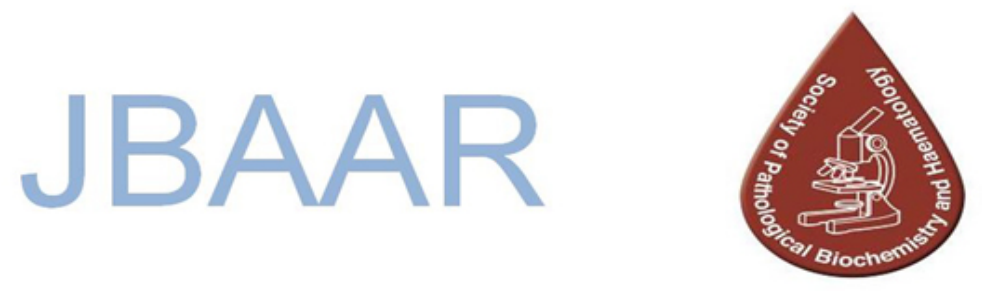

WWW.JBAAR.ORG

\title{
Protective effect of garlic on methyl ethyl ketone-induced biochemical changes in male rabbits
}

\author{
Zuhair Y. A1-Sahhaf \\ Department of Biology Faculty of Applied Sciences, Umm Al Qura \\ University, Makkah, Saudi Arabia \\ ( Corresponding author email: zyshhaf@uqu.edu.sa)
}

\begin{abstract}
The present study evaluated the effect of inhalation of methyl ethyl ketone (MEK) on biochemical parameters of rabbits and the possible protective role of garlic aqueous extract. Animals were divided into 4 groups. Group 1 served as controls, group2 given garlic aqueous extract (0.2mg/ml, for a week), group 3 were inhaled (MEK) (500 ppm for 3 minutes daily for 4 weeks), group 4 inhaled MEK for 4 weeks and given garlic for another week. The results showed that exposing animals to MEK induced significant decrease in RBCs count, hemoglobin, hematocrit percentage and blood platelets. On the other hand, the WBCs count was increased. Triglycerides, cholesterol and transaminases (ALT and AST) were increased in the sera of treated rabbits. Rabbits inhaled MEK for two weeks and given garlic aqueous extract for another week showed an improvement of the hematological and biochemical parameters. It is concluded from the present results that garlic aqueous extract modulated toxicity of MEK and this may be attributed to the antioxidant activity of garlic constituents
\end{abstract}

Keywords: MEK, Rabbits, Hematology, Transaminases, Garlic, Antioxidants

\section{Introduction}

Methyl ethyl ketone (MEK) is a colorless liquid solvent used in the manufacture of a number of resins, waxes, and coatings, as well as a general industrial solvent for nitrocellulose coating, vinyl film, and smokeless powder manufacture (ATSDR, 1992).
Exposure to MEK caused many hazardous effects. Initial symptoms of MEK inhalation include nausea, headache, dizziness, and respiratory distress. Over the next several days, the subject experienced impaired concentration, memory loss, tremor, gait ataxia, and dysarthria (Welch et al. 1991). Low level chronic exposure to MEK has been shown to cause decreased memory and impairment of the central nervous system (Perbellini et al.1984). Schwetz et al. (1991) reported that MEK has Embryo- and fetoxtoxicity in mice. Muttray et al. (2002) reported that exposing 19 healthy male volunteers to $200 \mathrm{ppm}$ (590 $\mathrm{mg} / \mathrm{m}^{3}$ ) MEK or filtered air for 4 hours in a crossover study design resulted in irritation of mucous membranes, difficulties in breathing, and pre-narcotic symptoms. A single oral dose of 1,082 mg/kg of MEK in corn oil induced renal tubule necrosis in F344 rats (Brown and Hewitt, 1984). Liver necrosis has been observed in both adult and pediatric patients who have experienced chronic exposure to MEK (Enckevort et al. 2008). Spirtas et al. (1991) reported a significantly increased standard mortality ratio for multiple myeloma among women exposed to MEK.

Many traditional plants are now used in treatment of diseases. Garlic, Allium sativum, is a member of the lily family that has been used as a food plant for over 10,000 years. Recent studies showed that garlic have anticancer, antioxidant, immunomodulatory, anti-inflammatory, hypoglycemic and hormone-like effects (Reuter et al. 2002). Garlic was also found to have hepatoprotective effect (Pal et al. 2006). Epidemiological findings a demonstrated an inverse relationship between garlic 
consumption and the incidence of stomach, colorectal and prostate cancer (You et al.1989, Hsing et al. 2002). Garlic also increases anti-inflammatory monocyte IL-10 production and decreases proinflammatorycytokines such as TNF- $\alpha$, IL-1 $\beta$, IL-6, IL-8, T cell interferon gamma, IL-2 (Hodge et al. 2002). The present work was designed to study the effect of garlic on MEK-induced biochemical changes in rabbits.

\section{Materials and Methods}

Adult male New Zealand rabbits weighing 500-700 g were used in the present experiments. The animals were housed in universal galvanized wire cages at room temperature $\left(22 \pm 2 \mathrm{C}^{\circ}\right)$ and in a photoperiod of 12:12 light/dark cycle, $50 \% \pm 5 \%$ humidity. They were acclimatized for 2 weeks prior to the start of the experiment. Animals were fed on pellets of standard rabbit ration and free access to water ad libitum. They were divided into four groups:

Group 1: animals of this group (4 rabbits) served as controls.

Group 2: At the $5^{\text {th }}$ week, animals of this group (4 rabbits) were orally given garlic aqueous extract at a dose level of $0.2 \mathrm{mg} / \mathrm{ml}$, for a week. Freshly prepared garlic homogenate was prepared by diluting $20 \mathrm{~g}$ of blended garlic with 1000 $\mathrm{ml}$ of distilled water.

Group 3: animals of this group (8 rabbits) were inhaled methyl ethyl ketone (MEK). Animals were kept individually in a closed cage and exposed to 500 ppm MEK for 3 minutes daily for 4 weeks and left without exposing to MEK for a week .

Group 4: animals of this group (8 rabbits) were inhaled methyl ethyl ketone (MEK) for 4 weeks and then given garlic aqueous extract for another week.

\section{Biochemical assays}

The treated animals and their controls were sacrificed by decapitation after 5 weeks of treatment. For hematological study, blood was collected from control and treated animals. The hematological parameters: red blood cells count ( RBCS), hemoglobin value (Hb), hematocrit value ( $\mathrm{HCT} \%$ ), white blood cells count (WBCS) and blood platelets number were measured by a fully automated Coulter counter (Coulter Electronics Limited, England). For biochemical assays, blood samples were collected from animals and sera were obtained by centrifugation of the blood sample and stored at $-20^{\circ} \mathrm{C}$. Triglycerides, cholesterol, aspartate aminotransferase (AST) and alanin aminotransferase (ALT) were measured using a fully automated Hitachi 911 analyzer (Tokyo, Japan). A commercial randox kits (Randox Laboratories, LTD, Ardomre, Crumlin, United Kingdom) were used in these analysis.

\section{Statistical analysis}

Data were expressed as mean values \pm SD and the criterion for statistical significance was set at $\mathrm{P}<0.05$. All statistical analyses were performed using SPSS statistical version 16 software package (SPSS ${ }^{\circledR} 4$ Inc., USA).

\section{Results}

\section{i.Hematological results}

Data in table 1 showed that treating rabbits with MEK for 4 weeks caused significant decrease in RBCs count and hemoglobin contents. Similarly, the mean corpuscle volume (MCV) and mean corpuscle hemoglobin content (MCHC) decreased in MEK-treated animals. The hematocrite percentage (HCT \%) decreased after treatment with MEK. On the other hand the mean WBCs count increased in rabbits-inhaled MEK. The mean number of platelets and mean platelet volume showed significant decrease in the treated animals. No significant changes were recorded between controls or animals treated with garlic. Animals inhaled MEK and given garlic showed normal values of all these hematological parameters.

\section{ii.Biochemical results}

Exposing animals to MEK resulted in significant increase in cholesterol compared with control group, and the mean values were $73 \pm 3.6,51 \pm 2.4$, respectively (Fig.1). Also, triglycerides increased significantly $(\mathrm{P}<0.05)$ in $\mathrm{MEK}$ exposed rabbits (Fig.2). The values of cholesterol and triglycerides decreased in animals treated with MEK and garlic. Concernning the change in transaminases, figure 3 showed that ALT increase in sera of animals exposed to MEK compared with controls and returned to normal value after treatment with MET and garlic (Fig.3). Similarly, AST increased in sera of rabbits treated with MEK and decreased in animals exposed to MEK and given garlic (Fig.4).

\section{Discussion}

MEK is used as a solvent in the application of protective coatings (varnishes) and adhesives (glues and cements), in magnetic tape production, in smokeless powder manufacture, in the dewaxing of lubricating oil, and in food processing. WHO (1992) estimated levels of daily MEK intake from different sources as follows: foodstuffs - 1,590 $\mu \mathrm{g} / \mathrm{day}$; drinking water (2 liters) - 3.2 $\mu \mathrm{g} /$ day; rural outdoor air -36 $\mu \mathrm{g} /$ day; urban outdoor air 760 $\mu \mathrm{g} /$ day; and tobacco smoke $1,620 \mu \mathrm{g} / \mathrm{day}$. The present work studied the biochemical effects of MEK in rabbits. The results showed that inhalation of MEK caused a significant decrease in RBCs count, haemoglobin content, hematocrite percentage and mean number of platelets. On the other hand, WBCs count increased. In agreement with these results, Gaunt et al. (1972) exposed CFE rats (15/sex/group) to 3-hydroxy-2-butanone (metabolite of MEK) in drinking water $(0,750,3,000$, or $12,000 \mathrm{ppm})$ for 13 weeks. Their results showed that in males and females hemoglobin decreased by 4.9 and $4.2 \%$ as compared to controls and red blood cell count decreased by 5.4 and $8.3 \%$ with corresponding increases in reticulocytes, respectively. 
Table (1) Effect of MEK and /or garlic on hematological parameters of male rabbits

\begin{tabular}{|c|c|c|c|c|}
\hline Parameters & $\begin{array}{c}\text { Contro } \\
1\end{array}$ & Garlic & MEK & $\begin{array}{l}\text { MEK+ } \\
\text { garlic }\end{array}$ \\
\hline $\begin{array}{r}\text { RBCs } \\
10^{6} / \mathrm{mm}^{3}\end{array}$ & $4.40 \pm 0.11$ & $4.36 \pm 013$ & $2.93 \pm 0.2^{*}$ & $4.07 \pm 0.4$ \\
\hline Hb gm/dl & $12.3 \pm 1.3$ & $11.6 \pm 1.1$ & $6.2 \pm 1.2 *$ & $9.5 \pm 1.4$ \\
\hline $\operatorname{MCV(fl)}$ & $73.9 \pm 5.4$ & $72.5 \pm 4.6$ & $65.9 \pm 2.6$ & $68.8 \pm 4.2$ \\
\hline MCHC & \begin{tabular}{|l|}
$37.5 \pm 3.1$ \\
\end{tabular} & \begin{tabular}{|l|}
$39.8 \pm 2.2$ \\
\end{tabular} & $30.5 \pm 1.4$ & $33.5 \pm 1.4$ \\
\hline НСТ \% & $32.5 \pm 2.3$ & $31.8 \pm 1.8$ & $21.3 \pm 1.7^{*}$ & $31.3 \pm 2.3$ \\
\hline $\begin{array}{l}\text { Platelets } \\
10^{3} / \mathrm{L}\end{array}$ & $502 \pm 10.1$ & $500 \pm 12.2$ & $344 \pm 8.3^{*}$ & $468 \pm 11.3$ \\
\hline MPV (fl) & \begin{tabular}{|l|l|}
$7.8 \pm 0.5$ \\
\end{tabular} & $7.6 \pm 1.1$ & $5.3 \pm 1.2 *$ & $6.7 \pm 1.3$ \\
\hline $\begin{array}{l}\text { WBCs10 } \\
3 / \mathrm{mm}^{3}\end{array}$ & $9.5 \pm 1.5$ & \begin{tabular}{|l}
$8.4 \pm 1.3$ \\
\end{tabular} & $13.1 \pm 1.5^{*}$ & $10.2 \pm 1.1$ \\
\hline
\end{tabular}

-Values are expressed as mean \pm SD

- (*) Significant at $\mathrm{p}<0.05$

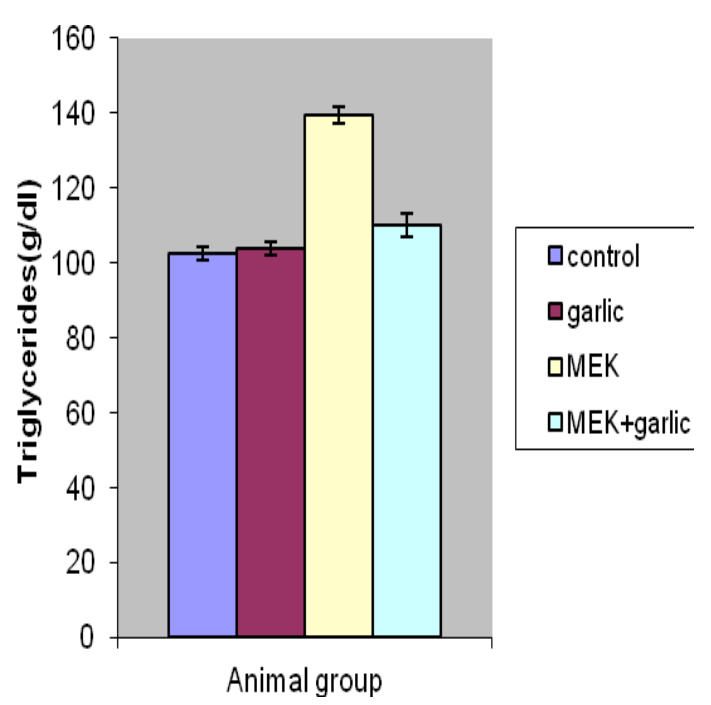

Fig.2. Effect of different treatments on triglycerides

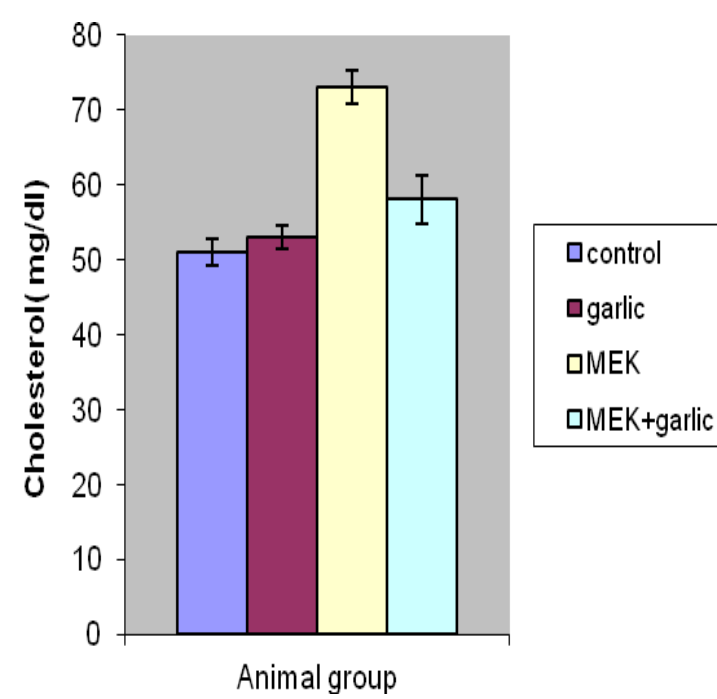

Fig.1. Effect of different treatments on cholesterol 


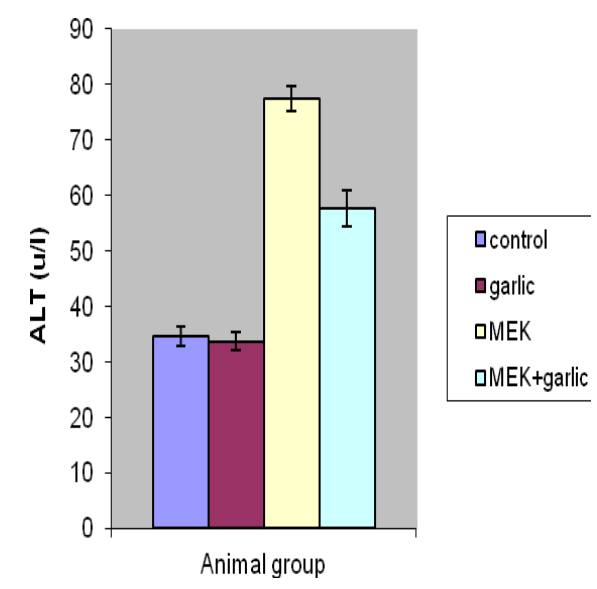

Fig.3. Effect of different treatments on ALT.

Abnormal rise in the levels of serum ALT, AST, has been observed in sera of animals exposed to MEK. The elevated levels of these biochemical markers in the serum are indicators of hepatotoxicity. Cavender et al. (1983) exposed male and female Fischer 344 rats (15/sex/group) in a whole body dynamic air flow chamber to MEK 6 hours/day, 5 days/week for 90 days. They showed that animals had a significant increase in liver weight and brain weight/body weight ratio, at necropsy. Serum glutamic-pyruvic transaminase (SGPT) activity in the $2500 \mathrm{ppm}$ female rats was elevated while the 5000 ppm female rats exhibited significantly decreased SGPT activity. In addition, alkaline phosphatase, potassium and glucose values for the 5000 ppm female rats were increased. A slight but not statistically significant decrease in number of red blood cells was recorded.

Involvement of oxidative stress following acute exposure to xenobiotics has been reported recently. Numerous studies indicate that toxic chemicals can cause oxidative stress by the generation of free radicals and induce lipid peroxidation. Free radical production and lipid peroxidation has been the suspected mechanism by which (methyl ethyl ketone peroxide) MEKP results in hepatotoxicity (Moon et al. 2010). The majority of MEK is oxidized by the cytochrome P450 monooxygenase system (P450IIE1 and IIB isozymes) to the primary metabolite,3-hydroxy-2-butanone (3H-2B), which is subsequently reduced to 2,3-butanediol (2,3-BD) (Brady et al., 1989). Thus the obtained results may be attributed to oxidative stress induced by MEK.

Regarding the effect of garlic, animals inhaled MEK for a week and given garlic for another week showed an improvement in the hematological and biochemical parameters. Several investigators studied the therapeutic effects of garlic and its extracts. Ahmed and Sharma (1997) reported that garlic caused a significant decrease in blood

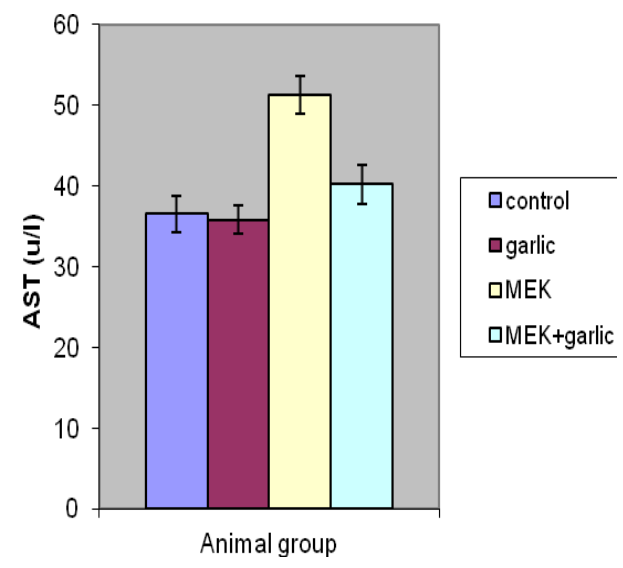

Fig.4. Effect of different treatments on AST.

glucose, serum total cholesterol and serum alkaline phosphatase in rats. Lipid peroxides, uric acid, blood glucose, total lipid, triglycerides and cholesterol were decreased in streptozotocin-induced diabetic rats fed on diet containing garlic (Mamdouh et al. 2003). Asaduzzaman et al. (2011) reported that garlic fractions reduced cholesterol, triglycerides, ALT and AST in alloxan-induced diabetic rats. Pal et al. (2006) reported that garlic extract prevent hepatotoxicity and elevation of ALT and AST in rats treated with isoniazid and rifampicin. In diabetic rats, elevated levels of glucose, cholesterol and triglycerides, the increment of the activities of ALT and AST, increased food and water consumption were decreased in rats given garlic extracts (Masjedi et al. 2013).

Most of the prophylactic and therapeutic effects of garlic are ascribed to its antioxidant activity. Garlic contains different antioxidant components. The biochemical constituents of garlic bulb are in following proportions containing approximately 65\% water, $28 \%$ carbohydrates (mainly fructans), 2.3\% organosulfur compounds, $2 \%$ protein (mainly alliinase), 1.2\% free amino acids (mainly arginine)and $1.5 \%$ fiber. A large amount of $\gamma$-glutamylcysteine is present in the garlic bulb (Amagase et al.2001). Munday and Munday (2004) reported that organosulfur compounds have antioxidant properties and these effects are related to their ability to inhibit phase I enzymes and induce phase II enzymes or bind to exogenous toxins through sulfhydryl groups. Borek (2001) reported that garlic extract increase antioxidant action by scavenging reactive oxygen species, enhancing the antioxidant enzymes superoxide dismutase, catalase and glutathione peroxidase, in the cells. Garlic has been shown to inhibit lipid peroxidation and dose-dependent induction of endogenous antioxidants in rat kidney and liver (Banerjee et al. 2001). Mostafa et al. (2013) reported that MDA levels showed significant reduction in the plasma and the activities of SOD and GSH-Px showed significant increase 
in erythrocytes of normal postmenopausal women after 8 weeks of garlic. It is concluded from the present results that garlic aqueous extract prevented toxicity of MEK and this may be attributed to the antioxidant activity of garlic constituents.

\section{References}

Ahmed RS, Sharma SB. (1997). Biochemical studies on combined effects of garlic (Allium sativum Linn) and ginger (Zingiber officinale Rosc) in albino rats. Indian J Exp Biol. 35(8):841-3.

Amagase H, Petesch BL, Matsuura H, Kasuga S, Itakura Y. (2001). Intake of garlic and its bioactive components. J Nutr. 131:955S-962S

Asaduzzaman, M., Afia Akhtar, M., Islam,A., Khan,R. Anisuzzaman, ASM, Ahmed, M. (2011). Evaluation of antidiabetic, antihyperlipidemic and hepatoprotective effects of Allium Sativum (Linn.) in Alloxan Induced Diabetic Rats. Bangladesh Pharm J.13(1): 28-33.

ATSDR (Agency for Toxic Substances and Disease Registry), (1992). Toxicological profile for 2-butanone. Atlanta, GA: U.S. Department of Health and Human Services, Public Health Service.

Banerjee SK, Maulik M, Manchanda SC, Dinda AK, Das TK, Maulik SK. (2001). Garlic-induced alteration in rat liver and kidney morphology and associated changes in endogenous antioxidant status. Food Chem Toxicol. 39: 793-797

Borek.C, (2001). Antioxidant Health Effects of Aged Garlic Extract. J. Nutr. 131: 1010-1015

Brady, JF; Li, D; Ishizaki, H. (1989). Induction of cytochromes P450IIE1 and P450IIB1 by secondary ketones and the role of P450IIE1 in chloroform metabolism. Toxicol Appl Pharmacol. 100:342-349.

Brown, EM, Hewitt, WR. (1984). Dose-response relationships in ketone-induced potentiation of chloroform hepato- and nephrotoxicity. Toxicol Appl Pharmacol. 76:437-53.

Cavender, FL, Casey, HW, Salem, H. (1983) A 90day vapor inhalation toxicity study of methyl ethyl ketone. Fundam Appl Toxicol. 3(4):264-70.

Enckevort, C C, Touw, DJ, Vleming.LJ. (2008). Nacetylcysteine and hemodialysis treatment of a severe case of methyl ethyl ketone peroxide intoxication. Clin. Toxicol. 46:74-78.

Gaunt, IF, Brantom, PG, Kiss, IS. (1972). Shortterm toxicity of acetoin (acetylmethylcarbinol) in rats. Food Cosmet Toxicol. 10(2):131-41.

Hodge G,Hodge S, Han P.(2002). Allium sativum (garlic) suppresses leukocyte in flammatory cytokine production in vitro: potential therapeutic use in the treatment of inflammatory bowel disease. Cytometry 48:209-215

Hsing AW, Chokkalingam AP, Gao YT.(2002). Allium vegetables and risk of prostate cancer: a populationbased study. J Natl Cancer Inst. 94:1648-51.
Mamdouh, MA., Abdel-Raheim, MA. (2003). Oxidative stress in streptozotocin-induced diabetic rats. Effects of garlic oil and melatonin. Comparative Biochem Physiol. Part A, 135: 539-547

Masjedi,F., Gol,A., Dabiri,S. (2013). Preventive Effect of Garlic (Allium sativum L.) on serum biochemical factors and histopathology of pancreas and liver in streptozotocin-induced diabetic rats. Iranian J Pharmaceutical Res. (3): 325-338

Moon, S., Lee, S. Choi,S.. Hong.Y. (2010). Gastric emphysema after methyl ethyl ketone peroxide ingestion. Clin Toxicol. 48 (1):90-91.

Mostafa, RM, Moustafa, YM., Mirghani, Z., AlKusayer, GM. and Moustafa, KM. (2013).Antioxidant effect of garlic (Allium sativum) and black seeds (Nigella sativa) in healthy postmenopausal women. SAGE Open Medicine, January December 2013; vol. 1, 2050312113517501

Munday R , Munday CM. (2004). Induction of phase II enzymes by aliphatic sulfides derived from garlic and onions: an overview. Methods Enzymol. 382 : 449-456

Muttray, A, Jung, D, Klimmek, L. (2002). Effects of an external exposure to $200 \mathrm{ppm}$ methyl ethyl ketone on nasal mucosa in healthy volunteers. Int Arch Occupat Environ Health 75(3):197-20.

Pal R, Vaiphei K, Sikander A, Singh K, Rana SV. (2006). Effect of garlic on isoniazid and rifampicin - induced he-patic injury in rats. World Gastroenterol; 12: 636-639.

Perbellini, L; Brugnone, F; Mozzo, P. (1984). Methyl ethyl ketone exposure in industrial workers. Uptake and kinetics. Int Arch Occup Environ Health 54:73-81.

Reuter HD, Koch HP , Lowson DL. (2002). Therapeutic effects and applications of garlic and its preparation. In: Garlic:The science and therapeutic application of Allium sativumL and related species, 2ed (Koch, HP and Lawson, DL. eds), William and Wilkins, Baltimore MB, pp 135 - 212.

Schwetz, BA; Mast, TJ; Weigel, RJ. (1991). Developmental toxicity of inhaled methyl ethyl ketone in mice. Fundam Appl Toxicol 16:742-8.

Spirtas, R, Stewart, PA, Lee, JS. (1991). Retrospective cohort mortality study of workers at an aircraft maintenance facility: I. Epidemiological results. II. Exposures and their assessment. Br J Ind Med 48(8):515-37.

Welch, L, Kirshner, H, Heath, A. (1991). Chronic neuropsychological and neurological impairment following acute exposure to a solvent mixture of toluene and methyl ethyl ketone (MEK). J Toxicol Clin Toxicol 29(4):435-45.

WHO (World Health Organization). (1992). Methyl ethyl ketone. Environmental Health Criteria. Volume 143.

You WC, Blot WJ, Chang YS.(1989). Allium vegetables and reduced risk of stomach cancer. J Natl Cancer Inst. 81:1624 